\title{
A Case Report on Staphylococcal Scaled Skin Syndrome
}

\author{
Kavya Nedamanuru1 ${ }^{*}$, Sai Jyothi Dampetla', Divya Gopineni ${ }^{2}$ \\ Pharm.D VI year ${ }^{1}$, Assistant Professor ${ }^{2}$, Department of Pharmacy Practice, Sri Padmavathi School of Pharmacy, Tiruchanoor, Tirupathi, \\ Andhra Pradesh, INDIA.
}

\begin{abstract}
Staphylococcal Scalded Skin Syndrome (SSSS) is the medical term used to define a skin condition induced by the exfoliative toxins produced by Staphylococcus aureus. It is also called as Ritter disease, Lyell disease and staphylococcal necrolysis of epidermis. SSSS is most common in children and neonates, and it has been described in adults with renal failure, immunologic deficiency, and other chronic illnesses. The epidermolytic toxin causes loss of cell adhesion in the epidermis causing skin damage.In this article, we present a case of 32 days old male child with dark brown coloured macules all over the body. Based on clinical and paraclinical examinations a diagnosis of Staphylococcal scalded skin syndrome was established which responded favourably to antibiotic treatment, hydro-electrolytic re-equilibration, and adequate local hygiene.
\end{abstract}

Key words: Staphylococcal Scalded Skin Syndrome, Neonates, Erythematous lesions, Culture sensitivity test, Antibiotic therapy.

\section{INTRODUCTION}

Staphylococcal scaled skin syndrome is also called as Ritter disease, Lyell disease and staphylococcal necrolysis of epidermis is counted as one of the major skin infections. This disease generally occurs in new born and, in rare cases very old or the immunocomprimised, in which skin surface of large parts of body gets peeled off like burned skin by hot liquid. ${ }^{1}$ SSSS is caused by an exfoliative toxin produced by roughly $5 \%$ of Staphylococcal aureus. ${ }^{2}$ The two exfoliative or epidermolytic toxins responsible for the syndrome are named as ETA and ETB. The toxin causes loss of cell adhesion in the epidermis causing skin damage. ${ }^{3}$ The released toxins are biochemically serine proteases and are circulated from a localized source, leaving epidermal erosion at distant sites. It results into breakage of the desmoglobin 1 , which acts as adhesion molecule, breaking up the skin by inhibiting the adhesion of skin cells. The exfoliative toxin attacks on Desmoglobin, which is responsible for cellcell sticking leading to superficial lesion. ${ }^{4,5}$ Prodromal localised staphylococcal infection of the skin, throat, nose, mouth, umbilicus or GIT usually occurs. General malaise, fever, irritability, skin tenderness, facial edema, conjunctivitis may also be noted. The diagnosis of SSSS is by clinical examination and by collecting medical history. The oozed liquid or pus wrapped in cotton swab is used to identify the presence of the staphylococcal bacteria. The tests of skin biopsy and culture examination may also be performed to confirm the disease condition. ${ }^{6}$ Serological gel immunoprecipitation, radio immunological assays (RIA), enzyme linked immunosorbent assays (ELISA), gene sequence detection by DNA hybridization and polymerase chain reaction (PCR) are recently developed detection system for exfoliative toxins of SSSS. ${ }^{7}$ The treatment includes the administration of anti-staphylococcal antibiotics, liquids, electrolytes and the local treatment of the denudated areas.

\section{CASE REPORT}

A 37 days old neonate was admitted with complaints of fever and decreased acceptance of feeds from two days. The child was a full-term, normal baby.
DOI: 10.5530/ijopp.11.2.21

Address for correspondence: Kavya Nedamanuru Pharm.D VI year, Department of Pharmacy practice, Sri Padmavathi School of Pharmacy, Tiruchanoor, Tirupathi, Andhra Pradesh-517502, INDIA. Phone no: 7097533540 Email Id: kavyanedamanuru@ gmail.com

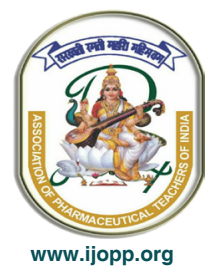


The child developed an erythematous rash on the 3rd day of admission to hospital. BCG vaccine was given and no history of similar complaints in the family. The fluid filled lesions started over the chest and spread to other parts of the body i.e. both upper and lower limbs within the next day. There was no history of administration of any drugs after birth. On examination, during admission, the child was febrile with erythematous lesions around the chest, hands, back and legs. After few days, peeling of skin and dark brown coloured macules over legs and arms were observed. A clinical diagnosis of SSSS was made. A culture sensitivity was performed which yielded a pure growth of Staphylococcal aureus. The child was treated with intravenous antibiotics like Amikacin, Cefotaxime and Amoxyclav. Isolyte P and Mupirocin ointment was also supplemented. The child was completely treated after 1 week of hospital admission.

\section{DISCUSSION}

The SSSS is not a very common disease throughout world. The first noticed outbreak of SSSS was in Ireland. The danger of SSSS lies in the fact that a different type of bacteria may invade some areas of the skin and may culminate complicated blood stream infection. Loss of body fluid occurs when skin peels away, and the residual layer dries out; fluid loss is a critical issue currently. The skin is the largest organ in the human body containing five sub layers. Below to the epidermis, a layer of dermis exists which is comprised of some tissues and acts to cushion the dermis from stress. Desmosome is a cell organelle which is localized and spot-like structure responsible for adhesion among cells. ${ }^{8}$ The main action of desmosomes is to resist shearing forces and is found in simple and stratified squamous epithelium. The exfoliative toxin attacks on Desmoglobin, which is responsible for cell-cell sticking leading to superficial lesion. Other complications include poor temperature control in young infants, septicaemia, guttae psoriasis, post streptococcal glomerulonephritis, boils, dermatitis, scabies, and diabetic ulcers. ${ }^{9}$ In order to prevent SSSS, several facts are to be considered which includes usage of antibacterial/ antiseptic soap for washing hands before touching the lesions. Schools and childcare centres are to be avoided when the infection is in contagious form. ${ }^{10}$ In the case reported, the characteristic skin lesions and failure to isolate Staphylococcus aureus from the lesions, but from blood culture staphylococcus aureus was isolated, absence of any mucosal lesions, and no history of administration of drugs was suggestive of SSSS. With prompt treatment the progression of the lesions was arrested, and no new lesions occurred. In the case described the child responded to injection
Amikacin and Cefotaxime with no new lesions and became apyrexic within 1 week.

\section{CONCLUSION}

SSSS occurs almost exclusively in infants and children under the age of 6 . It rarely occurs in older people except for those with kidney failure or a weakened immune system. Like other staphylococcal infections, SSSS is contagious. With prompt diagnosis and treatment, SSSS rarely causes death. SSSS is treated with oral antibiotics (may be given by IV in severe cases). The area of skin surface requires cleansing and dressings with antiseptic cream. Emergency medical treatment is required in condition of fluid or salt imbalance, so, early diagnosis should be done to prevent the mortality associated with SSSS complications.

\section{ABBREVIATIONS}

SSSS: staphylococcal scaled skin syndrome; ETA: Epidermolytic Toxin A; ETB: Epidermolytic toxin B; GIT : Gastrointestinal tract; RIA: Radio immunological assay

ELISA: Enzyme Linked Immunosorbent Assay; PCR: Polymerase chain reaction; BCG: Bacillus Calmette Guerin; IV: Intravenous

\section{CONFLICT OF INTEREST}

The authors declare no conflict of interest.

\section{REFERENCES}

1. Itani O, Crump R, Mimouni F, et al. Picture of the month: SSSS. Am J Dis Child 1992;146(4):425-6.

2. Adhisivam B, Mahadevan S. Abscess of the nasal septum with staphylococcal scalded skin syndrome. Indian Pediatr. 2006;43(4):372-3.

3. Krieg T, Bickers DR, Miyachi Y. Therapy of skin diseases: A worldwide perspective on therapeutic approaches and their molecular basis. London, New York: Springer Heidelberg Dordrecht.2010;18(4):.

4. Hanakawa Y, Schechter NM, Lin C, et al. Molecular mechanisms of blister formation in bullous impetigo and staphylococcal scalded skin syndrome. J Clin Invest. 2002;110(1):53-60.

5. Amagai M, Matsuyoshi N, Wang ZH, Andl C, Stanley JR. Toxin in bullous impetigo and staphylococcal scalded-skin syndrome targets desmoglein 1. Nat Med. 2000;6(11):1275-7.

6. Handler MZ, Schwartz RA. Staphylococcal scalded skin syndrome: diagnosis and management in children and adults. J Eur Acad Dermatol Venereol. 2014;28(11):1418-23. Foot C, Fraser JF. Uroscopic Rainbow: Modern Matula medicine. Postgrad Med J. 2006;82(964):126-9.

7. Mehrotra M, Wang G, Johnson WM. Multiplex PCR for detection of genes for Staphylococcus aureus enterotoxins, exfoliative toxins, toxic shock syndrome toxin 1, and methicillin resistance. J Clin Microbiol. 2000;38(3):1032-5.

8. Runswick SK, O'Hare MJ, Jones L, Streuli CH, Garrod DR. Desmosomal adhesion regulates epithelial morphogenesis and cell positioning. Nat Cell Biol. 2001;3(9):823-30.

9. Hoffmann R, Lohner M, Böhm N, Schaefer HE, Leititis J. Staphylococcal scalded skin syndrome (SSSS) and consecutive septicaemia in a preterm infant. Pathol Res Pract. 1994;190(1):77-81.

10. Kapral FA. Staphylococcus aureus: Some host-parasite interactions. Ann N Y Acad Sci.1974;236(1):267-76.

Indian Journal of Pharmacy Practice, Vol 11, Issue 2, Apr-Jun, 2018 